Supporting Information for:

\title{
Reprogrammable Three-Dimensional Configurations Using Ionomer Bilayers
}

\author{
Seonwoo Hwang, ${ }^{\dagger}{ }^{\dagger}$ Yecheol Rho, ${ }^{\S}$ Seog-Jin Jeon,,${ }^{*} \|$ Tae-Ho Kim,,${ }^{\dagger}$ Jang Yong Lee, ${ }^{\dagger}$ Young \\ Taik Hong, ${ }^{*} \dagger$ Soonyong So ${ }^{*}, \dagger$ \\ ${ }^{\dagger}$ Membrane Research Center and ${ }^{\S}$ Chemical Analysis Center, Korea Research Institute of \\ Chemical Technology, Daejeon 34114, South Korea \\ \$Department of Polymer Engineering, Chungnam National University, Daejeon 34134, South \\ Korea \\ "Department of Polymer Science and Engineering, Kumoh National Institute of Technology, \\ Gumi 39177, South Korea
}

\section{AUTHOR INFORMATION \\ Corresponding Authors}

Soonyong So

*E-mail: syso@krict.re.kr (S.S).

Seog-Jin Jeon

*E-mail: sjjeon@kumoh.ac.kr (S.-J.J).

Young Taik Hong

*E-mail: ythong@ krict.re.kr (Y.T.H). 
Dimensionless values. The equilibrium configurations of helices were evaluated by the following equations.

$$
\begin{gathered}
\tilde{p}=k_{0} \cdot p(1-v), \\
\tilde{w}=w \sqrt{k_{0}(1-v) / h,} \\
\tilde{r}=r \cdot k_{0}(1-v),
\end{gathered}
$$

which were evaluated with the preferred curvature, $k_{0}=\left(r_{\text {wide }}(1-v)\right)^{-1}$ (where $r_{\text {wide }}$ is the radius of cylindrical helices from wide strips), and measured pitches $(p)$, radii ( $r$ ) of helices with $w$, thickness $(h)$, and the Poisson's ratio (v) of Nafion membranes.

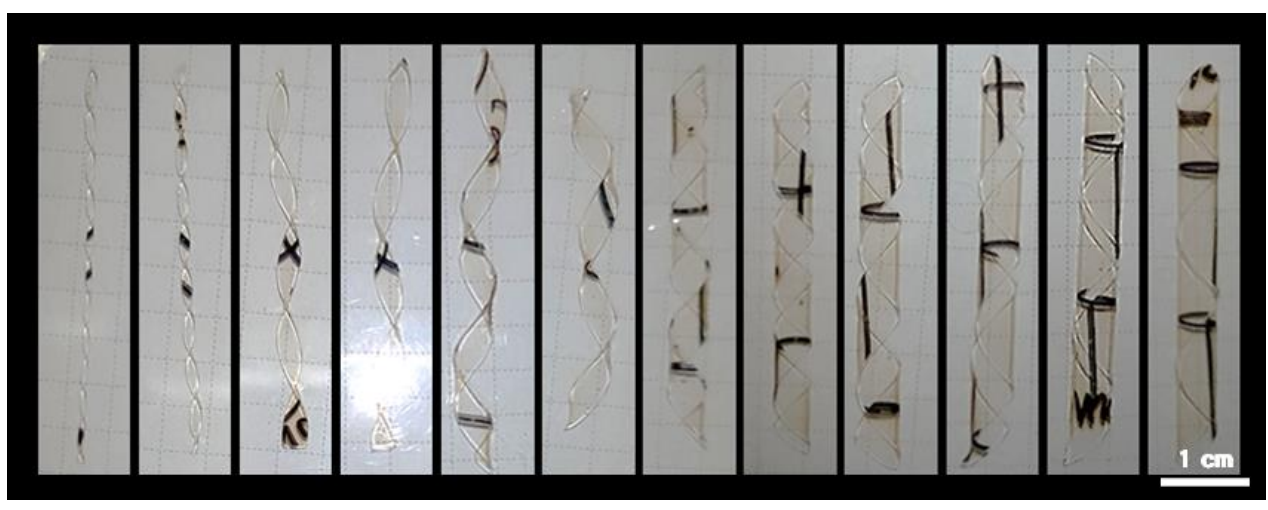

Figure S1. The equilibrium configurations of $\mathrm{H}-\mathrm{N} 1.94$ with $\theta=45^{\circ}$, and $w=1,1.5,2,2.5,3,3.5$, $4,5,6,7,8$, and $9 \mathrm{~mm}$ in water, respectively. 


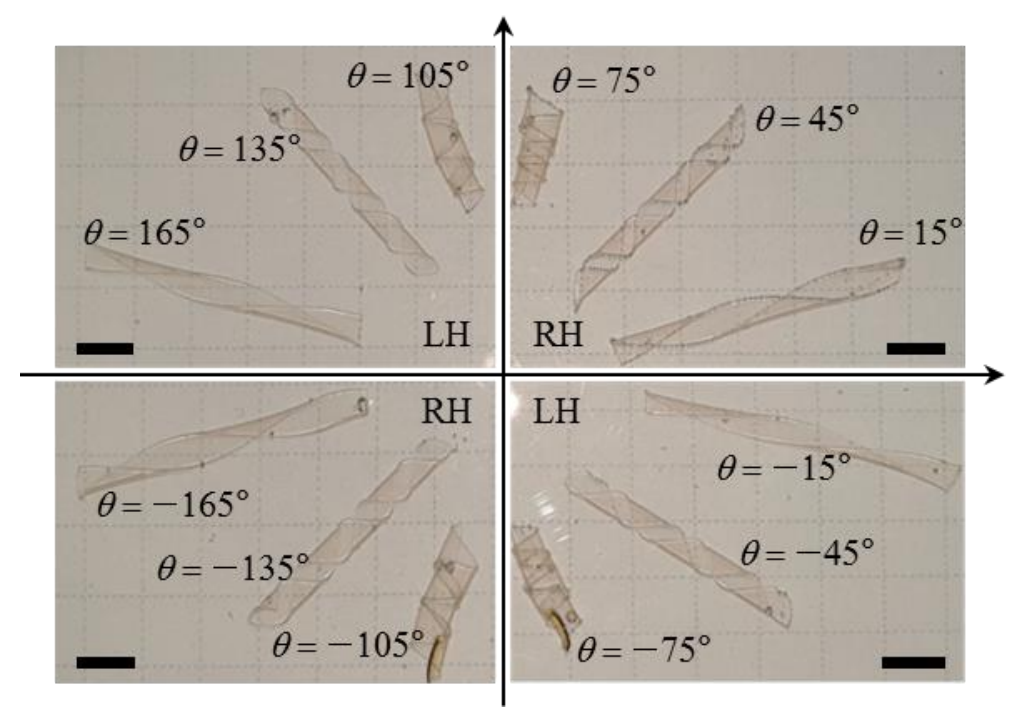

Figure S2. The equilibrium configurations of H-N3.47 (bottom swelling) at various cutting angles with $\widetilde{w}=4.57$ in water. Scale bars are $5 \mathrm{~mm}$.

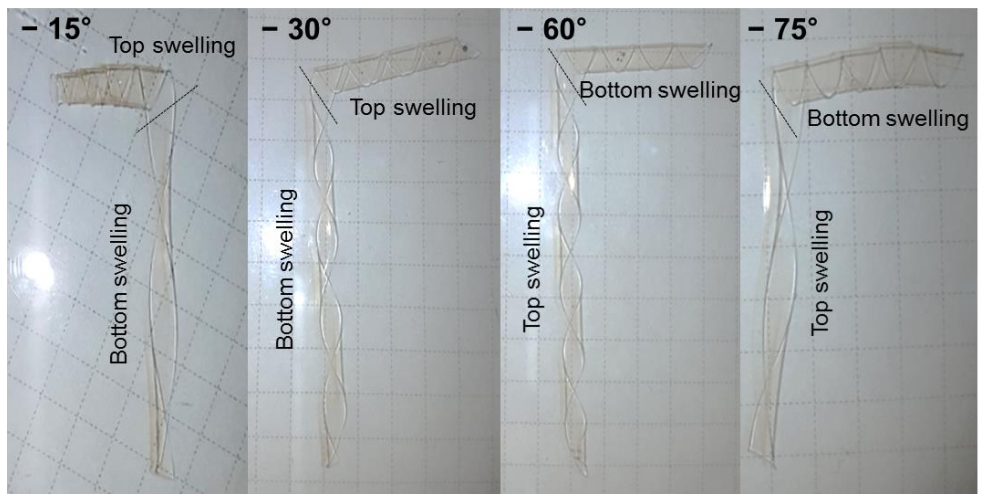

Figure S3. Bistable configurations of H-N2.98 strips $(w=5 \mathrm{~mm})$ depending on the swelling directions with various cutting angles $\left(-15^{\circ},-30^{\circ},-60^{\circ},-75^{\circ}\right)$. 
(a)

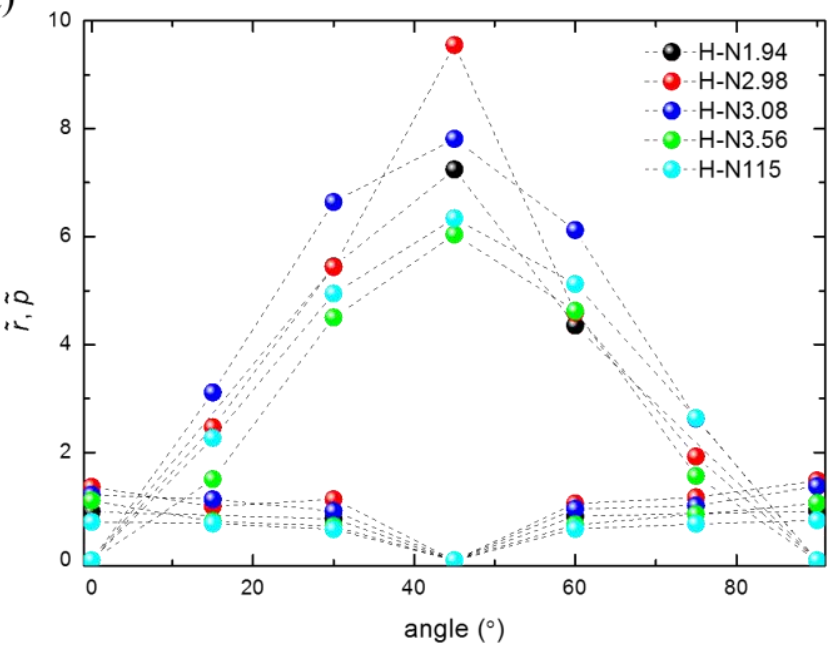

(b)

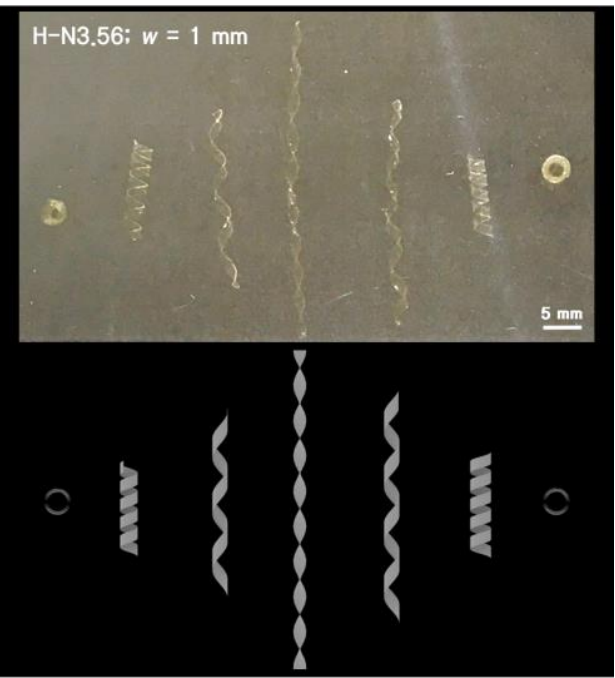

Figure S4. (a) Dimensionless pitch $(\tilde{p})$, and radius $(\tilde{r})$ of narrow $\left(\widetilde{w} \ll \widetilde{w}_{\mathrm{c}}\right)$ hot-pressed PFSA membranes as a function of the cutting angle $(\theta)$ from $0^{\circ}$ to $90^{\circ}$. (b) Configurations of narrow $(w=$ $1 \mathrm{~mm}) \mathrm{H}-\mathrm{N} 3.56$ strips $\left(\theta=0,15,30,45,60,75\right.$, and $\left.90^{\circ}\right)$ after swelling bottom layers first. Scale bars are $5 \mathrm{~mm}$.
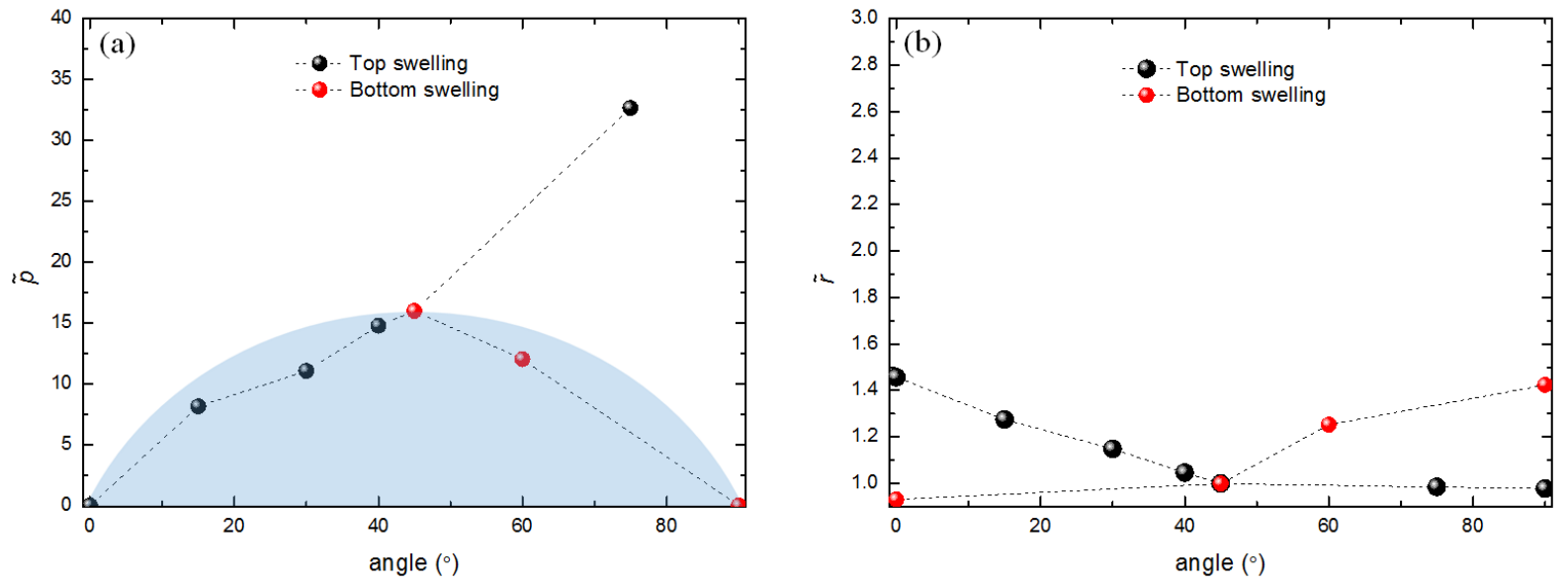

Figure S5. (a) Dimensionless pitch $(\tilde{p})$, and (b) radius $(\tilde{r})$ of wide ( $w=8 \mathrm{~mm}) \mathrm{H}-\mathrm{N} 2.86$ strips depending on the cutting angle $(\theta)$ from $0^{\circ}$ to $90^{\circ}$, and the first swelling layer. 

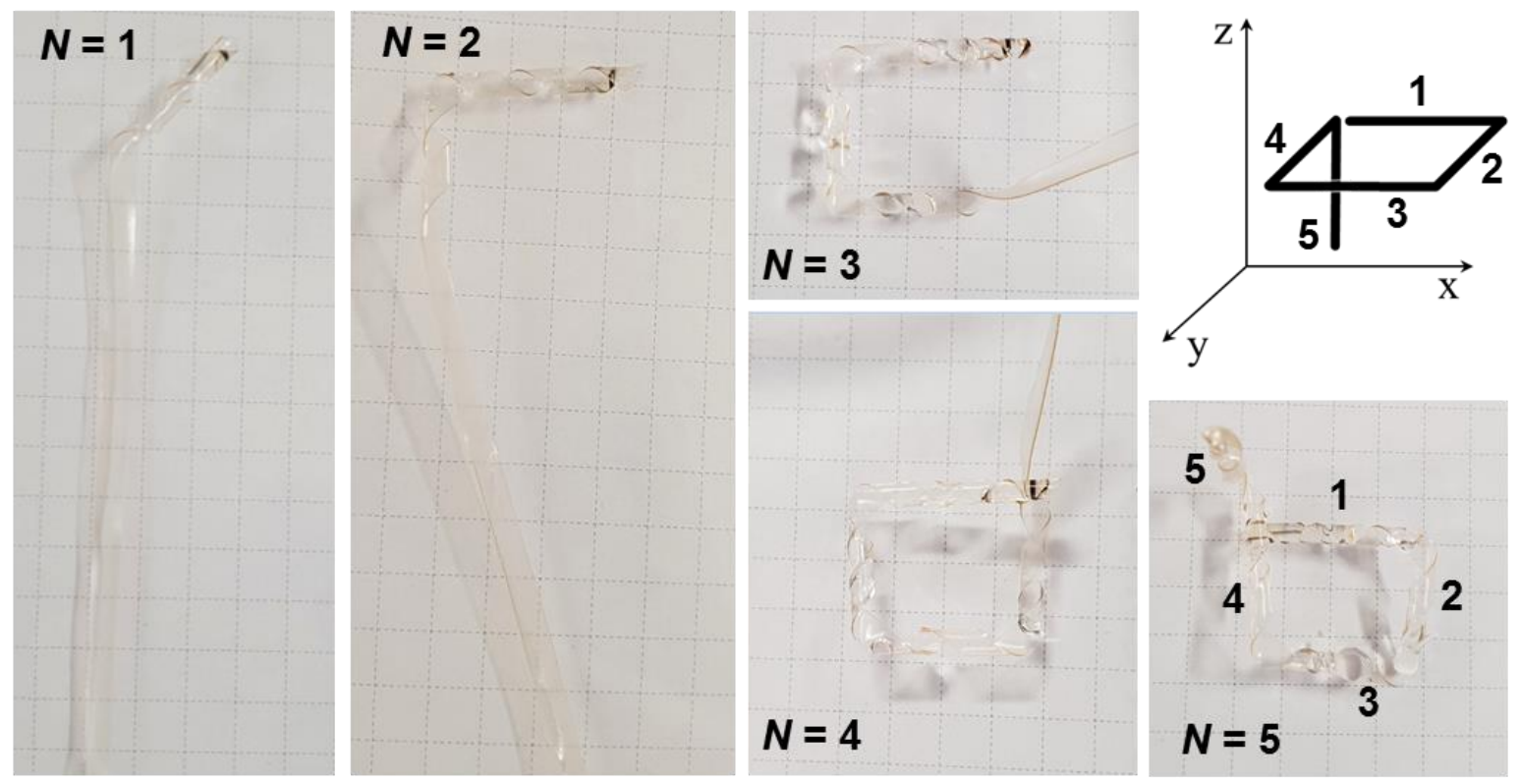

Figure S6. Construction procedure for a 3D structure with $\mathrm{H}-\mathrm{N} 3.47(w=3 \mathrm{~mm}, l=50 \mathrm{~mm}, \theta=$ $45^{\circ}$ ) from $N=1$ to $N=5$.
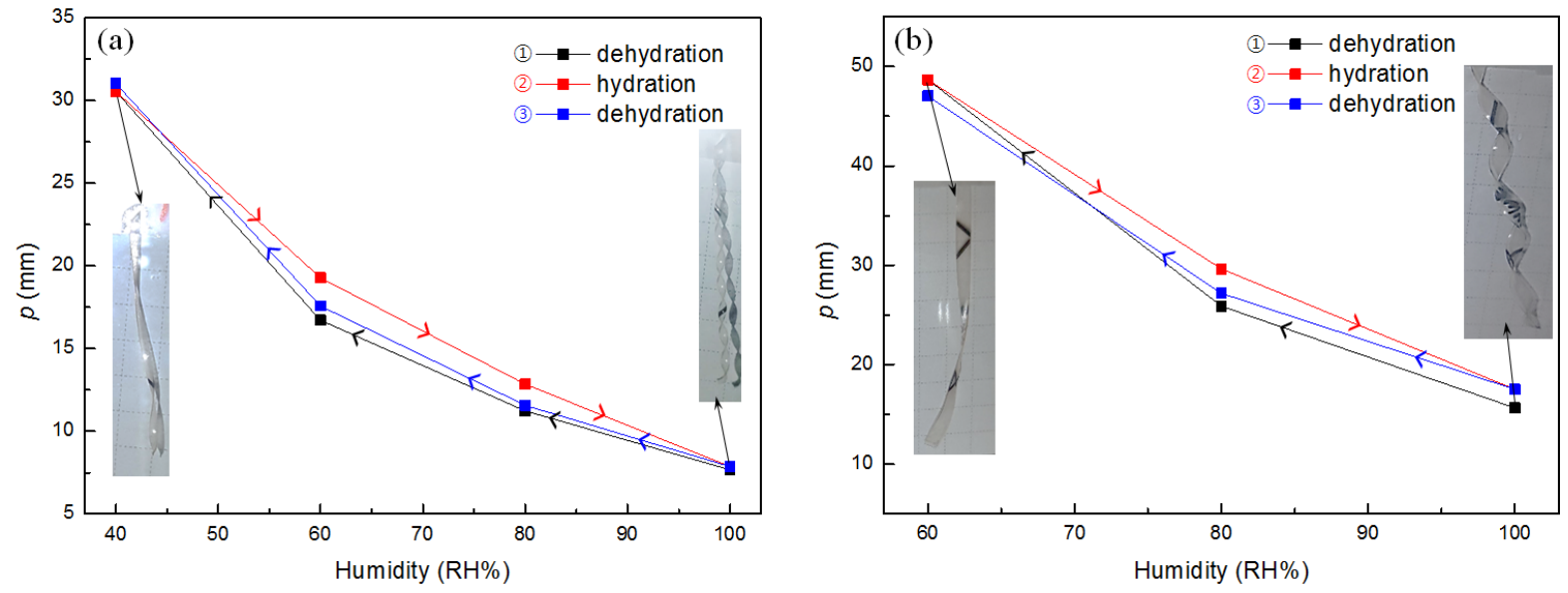

Figure S7. Humidity responsive actuations of (a) a twisted-helix of H-N3.56 ( $w=1 \mathrm{~mm}$ ), and (b) a cylindrical-helix of H-N1.94 $(w=3 \mathrm{~mm})$ during dehydration, hydration, and dehydration cycles at $30{ }^{\circ} \mathrm{C}$ in a humidity chamber. 

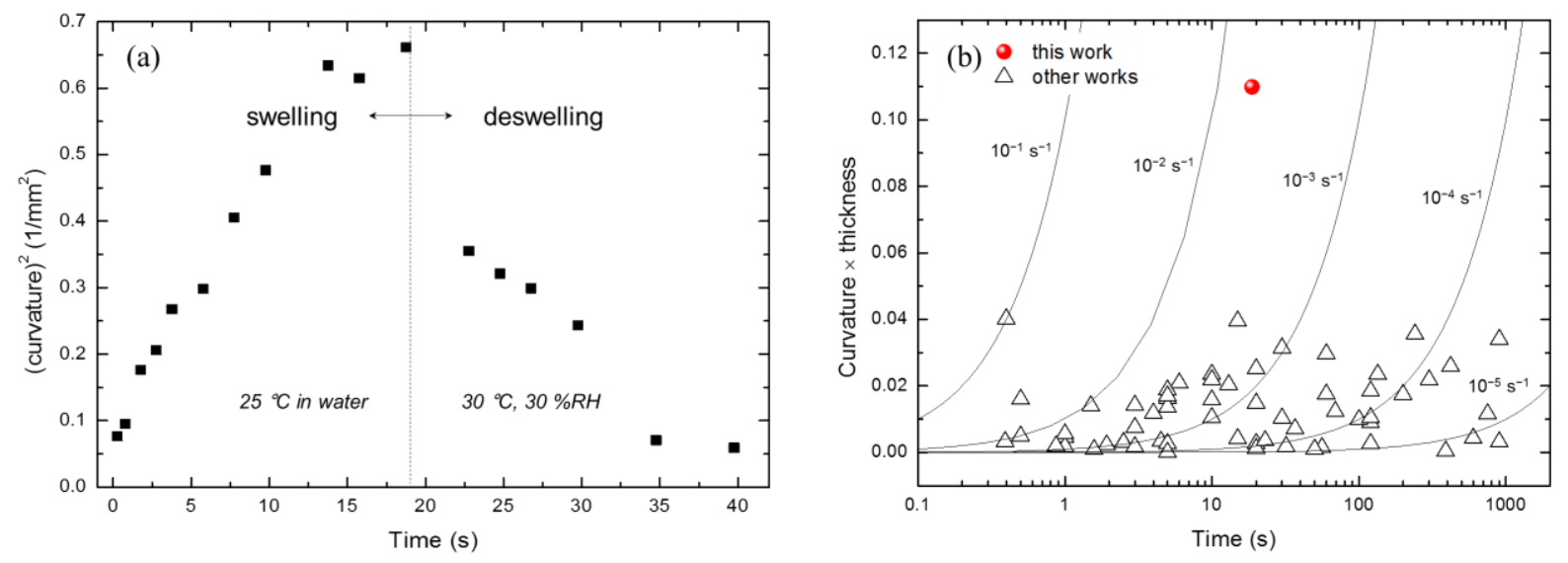

Figure S8. (a) Curvature of an $\mathrm{H}-\mathrm{N} 3.56\left(w=2 \mathrm{~mm}, l=18 \mathrm{~mm}, \theta=90^{\circ}\right)$ strip over time in water (swelling), and in a humidity chamber at $30{ }^{\circ} \mathrm{C}, 30 \% \mathrm{RH}$ (deswelling). (b) Plot of (curvature $\times$ thickness) and actuation time. Solid lines ( $k h / t$, where $k$ is the curvature, $h$ is the thickness, and $t$ is the actuation time) represent constant actuation speeds (solid lines) from $10^{-5}$ to $10^{-1} \mathrm{~s}^{-1}$. The result of this study (red circle) is compared with literature results (triangles) from Zhao et al. used in the previous study (reproduced with the permission from ref. S1. Copyright 2014 Springer Nature), and other recent studies. ${ }^{\mathrm{S} 2-\mathrm{S} 5}$ 


\section{REFERENCES}

(S1) Zhao, Q.; Dunlop, J. W. C.; Qiu, X.; Huang, F.; Zhang, Z.; Heyda, J.; Dzubiella, J.; Antonietti, M.; Yuan, J. An Instant Multi-Responsive Porous Polymer Actuator Driven by Solvent Molecule Sorption. Nat. Commun. 2014, 5, 4293.

(S2) Sun, J. K.; Lin, H. J.; Zhang, W. Y.; Gao, M. R.; Antonietti, M.; Yuan, J. A Tale of Two

Membranes: From Poly (Ionic Liquid) to Metal-Organic Framework Hybrid Nanoporous Membranes: Via Pseudomorphic Replacement. Mater. Horizons 2017, 4, 681-687.

(S3) Deng, J.; Li, J.; Chen, P.; Fang, X.; Sun, X.; Jiang, Y.; Weng, W.; Wang, B.; Peng, H. Tunable Photothermal Actuators Based on a Pre-Programmed Aligned Nanostructure. J. Am. Chem. Soc. 2016, 138, 225-230.

(S4) Zhao, Z.; Kuang, X.; Yuan, C.; Qi, H. J.; Fang, D. Hydrophilic/Hydrophobic Composite Shape-Shifting Structures. ACS Appl. Mater. Interfaces 2018, 10, 19932-19939.

(S5) Liu, Y.; Xu, B.; Sun, S.; Wei, J.; Wu, L.; Yu, Y. Humidity- and Photo-Induced Mechanical Actuation of Cross-Linked Liquid Crystal Polymers. Adv. Mater. 2017, 29, 1604792. 